\title{
Re-Aktiviert Bürgerschaftliches Engagement?
}

In den letzten Jahrzehnten hat das Bürgerschaftliche Engagement nicht nur an öffentlicher Beachtung gewonnen, sondern ist sogar als eine Art Re-Aktivierungsmittel gegen die Krise der Arbeitsgesellschaft, beim Umbau des Sozialstaats und für die Motivierung demokratischer Partizipation empfohlen worden. Unser Beitrag geht der Frage nach, ob und gegebenenfalls welche Aktivierungspotenziale von der Ausübung eines Bürgerschaftlichen Engagements auf diskontinuierlicher gewordene Erwerbsbiografien ausgehen können, oder ob es sich umgekehrt um bloße Aktivierungsrhetorik handelt.

\section{Das Ausgangsproblem}

In den letzten zwanzig Jahren bestimmen drei Krisendiagnosen die öffentliche Berichterstattung wie auch die sozial- und politikwissenschaftlichen Debatten. Die erste Problematik besteht im Wandel der wirtschaftlichen Strukturen westlicher Industrienationen, der zu einer Transformation und Krise der „Arbeitsgesellschaft“ geführt habe. Sichtbar sind die Veränderungen vor allem in den stetig hohen Arbeitslosenquoten seit Anfang der 1980er Jahre. Damit einher geht ein zweiter gesellschaftlicher Umbau, der auch als „Krise des Sozialstaats" bekannt ist. Die hohen Arbeitslosenzahlen führen zu einer erheblichen finanziellen Belastung der „sozialstaatlichen Kassen". Verstärkt werden diese finanziellen Belastungen durch den demografischen Wandel in Form einer alternden Gesellschaft. Parallel dazu wird ein dritter Prozess beobachtet: Die in manchen gesellschaftlichen Kontexten (insbesondere in politischen Parteien und Gewerkschaften) stark zurückgehende Bereitschaft der Bürger, sich aktiv zu beteiligen. Dies wird als „Krise der Demokratie“ registriert.

Alle drei Krisen können als Tendenzen der „De-Aktivierung“ von Bevölkerungsgruppen gelesen werden, die wiederum auch plötzliche Mangelerscheinungen hervorrufen könnten: Fehlende Fachkräfte, rückläufige politische Partizipation, sinkendes Engagement im sozialen Sektor.

Als Gegenmittel ist das „Bürgerschaftliche Engagement" in verschiedenen Facetten ins Spiel gebracht worden: Mal ist von Freiwilligenarbeit, dann vom Ehrenamt und vom sozialen oder vom bürgerschaftlichen Engagement die Rede. Und auch die Vielzahl von Untersuchungen, die es zur quantitativen Verbreitung des Bürgerschaftlichen Engagements gibt, kommt zu höchst unterschiedlichen Befunden und Szenarien. Während etwa der „Freiwilligensurvey“ für 1999 und 2004 (Rosenbladt 2000; Gensicke et al. 2006) eine Engagementquote von jeweils etwa einem Drittel der deutschen Wohnbevölkerung über 14 Jahren feststellte, kommt Dathe (2005, S. 474) auf der Grundlage des Sozio-oekonomischen Panels (SOEP) für den etwa gleichen Zeitraum zu einer Quote von etwa $16 \%$ der über 16-Jährigen. Auch mit Blick auf den European Value Survey (Halman et al.2006) kommt es zu solchen eklatanten Abweichungen.

Es fragt sich also, welchen gesellschaftlichen Stellenwert das Phänomen des Bürgerschaftlichen Engagements hat. Geht es um bloße „Aktivierungsrhetorik“, wie sie sich in den Diskursen um die „Neuerfindung des Sozialen“ (Lessenich 2008) mittlerweile ausbreitet? Oder könnten - zugespitzt formuliert - die Subjekte, die durch "Arbeitslosigkeitsökonomie" gesellschaftlich de-aktiviert wurden, über Bürgerschaftliches Engagement für den Arbeitsmarkt re-aktiviert werden?

In unserem Beitrag wollen wir genau dieser Frage nach möglichen Passungen, Schnittmengen und Übergängen zwischen Bürgerschaftlichen Engagement und (fehlender) Erwerbsarbeit und mangelndem Politikvertrauen nachgehen. Dabei beschäftigen wir uns zunächst konzeptionell und anhand der vorliegenden Forschungsliteratur mit Passungen in zwei Dimensionen: der Seite der gesellschaftlichen und organisatorischen Bedarfe an Erwerbsarbeit bzw. Bürgerschaftlichem Engagement und der Seite der individuellen Motivationsund Gelegenheitsstrukturen von Erwerbsarbeit und Bürgerschaftlichem Engagement (Abschnitt 2). Anhand von biografischem Datenmaterial aus einer empiri- schen Studie zum Bürgerschaftlichen Engagement widmen wir uns dann den besonderen Weisen der gesellschaftlichen ReAktivierung am Beispiel der ostdeutschen Transformationsgesellschaft (Abschnitt 3). Abschließend kommen wir nochmals auf die Frage der Aktivierungspotenziale des Bürgerschaftlichen Engagements zurück (Abschnitt 4).

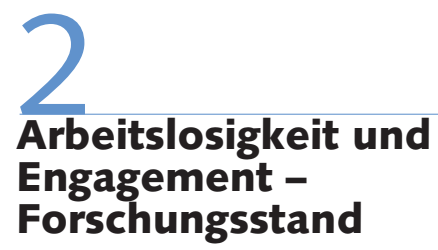

\subsection{STRUKTURDIFFERENZEN}

Gegen die Möglichkeit von faktisch in relevanter Zahl auftretenden Schnittmengen zwischen Erwerbsarbeit und Bürgerengagement sprechen schon konzeptionelltheoretische Gründe, die auf der organisatorischen und funktionsspezifischen Ebene angesiedelt sind. Demnach ist der Bereich der Erwerbsarbeit funktional und organisatorisch der Ökonomie und der Bereich des Bürgerengagements eher der politischen Kultur und dem öffentlichen Leben zuzuordnen. Insbesondere dann, wenn Bürgerschaftliches Engagement vom „Demokratiediskurs“ aus betrachtet wird, zei-

Michael Corsten, Prof. Dr., lehrt Soziologie
an der Universität Hildesheim. Arbeits-
schwerpunkte: Lebenslauf- und Generations-
forschung, Bildungssoziologie, Kultursozio-
logie.
e-mail: corsten@uni-hildesheim.de.
Romy Seidel, M. A. Soziologie, Koordina-
torin des "Mehrgenerationenhaus Jena“.
e-mail: Romeny@gmx.de.


gen sich kaum Synergien. So stellen Keupp et al. (2000, S. 217) lakonisch fest: „Bürgerschaftliches Engagement kann nicht als Modell der großräumigen Beschäftigungstherapie für jene Teile der Bevölkerung gelten, die als Problemgruppen des Arbeitsmarktes angesehen werden." Allerdings konstatieren die Autoren mögliche Verbindungslinien über den „Gemeinwohldiskurs“ und den „Diskurs sozialer Sicherung" (ebd.).

Das bedeutet aber zunächst einmal, dass von der funktionalen Grundausrichtung her Erwerbsarbeit und Bürgerschaftliches Engagement unterschiedlichen Organisationsmechanismen folgen. Erwerbsarbeit ist dem Prinzip der Wirtschaftlichkeit und daher organisatorischer Effizienz im Sinn von hoher formaler Rationalität (Rentabilitätskriterium) unterworfen. Da in Unternehmen Arbeitsplätze ein Kostenfaktor sind, müssen die dort Beschäftigten über Kompetenzen verfügen, die sich als effizientes Mittel zur Steigerung des Organisationsoutput einsetzen lassen. Eine solche Orientierung ist in den Bereichen der Politik, der Öffentlichkeit und der Kultur dem originären Bereich des Bürgerengagements - jedoch keineswegs zentral oder naheliegend. Dort geht es um die Organisation von Zustimmung und Beteiligung das politische, öffentliche und kulturelle Leben gewinnt seine „Lebendigkeit“ über die Herstellung des Mitmachens breiter Bevölkerungsschichten. So gesehen sind Erwerbsarbeit und Bürgerschaftliches Engagement sogar diametral entgegengesetzt. Unternehmen tendieren aus Gründen der Steigerung ihrer Organisationsrationalität $\mathrm{zu}$ einer Exklusionstendenz gegenüber dem Personal, indem sie Nicht-Beteiligung (mangelnde Leistungsbereitschaft) durch Ausschluss (Kündigung) sanktionieren. Politische Organisationen dagegen müssen um Teilhabe und Zustimmung umgekehrt kämpfen, um ihre Fähigkeit zur Regierungsbildung nicht zu gefährden. Und ähnlich müssen Einrichtungen des öffentlichen und kulturellen Raums um Personen werben, um ihren Bestand über hohe Teilnehmerquoten rechtfertigen zu können. Kurz gesagt: Wenn in der Sphäre des Erwerbslebens Exklusion als legitime Option besteht, ist sie im öffentlichen, politischen und kulturellen Raum moderner Demokratien geradezu illegitim.

Nun ließe sich einwenden, dass es in den Bereichen des Gemeinwohls und der sozialen Sicherung faktisch zu Aufwei-

Abb. 1: Engagementquoten im europäischen Vergleich - in \% -

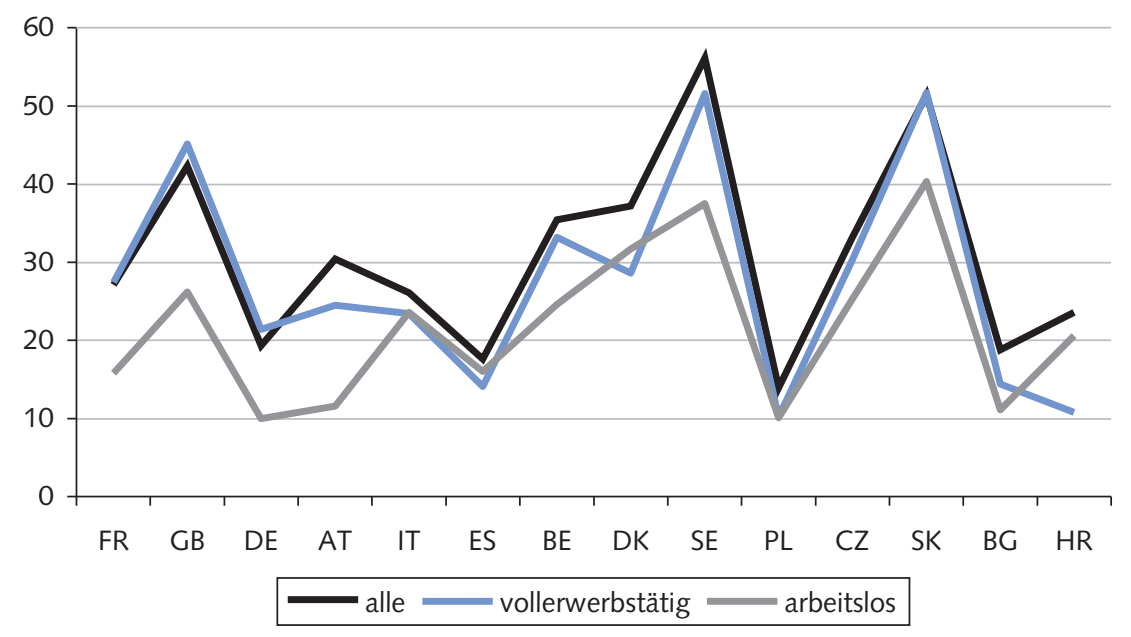

Quelle: European Value Survey 1999/2000; Berechnungen der Autoren.

WSI MITTEILUNGEN

chungen dieser scharfen Entgegensetzung kommt, insbesondere wenn wir an Beispiele liberal verfasster, meist angelsächsischer Wohlfahrtsstaaten denken. Hier weisen etwa in den USA auch Organisationen im sozialen Sektor (insbesondere im Gesundheitswesen) deutlich höhere Anteile von Freiwilligenarbeit auf als etwa in Deutschland. Allerdings sind dort die Beteiligungschancen exklusiver (insbesondere über Qualifikation und Weiterbildung) geregelt und ein System von finanziellen Aufwandsentschädigungen etabliert. D.h. der Bereich der Freiwilligenarbeit im liberalen Wohlfahrtsstaat weist eine Reihe von Zügen der Erwerbsgesellschaft auf, die den zivilgesellschaftlichen Charakter dieser Engagementformen zurückdrängen. Auch in Deutschland finden wir in einigen Bereichen des Bürgerschaftlichen Engagements deutlichere Formen der Organisiertheit, die in Form von Aufwandsentschädigungen oder Qualifikationsvoraussetzungen, etwa im Sportbereich oder in sozialpsychologischen Kontexten ( $z$. B. Telefon- oder Notfallseelsorge), sichtbar werden.

Es stellt sich nun die Frage, ob solche organisatorischen Entwicklungen dazu führen, dass arbeitslose Personen in stärkerem Maße an Formen des Bürgerschaftlichen Engagements partizipieren. Damit befinden wir uns bei der individuellen Dimension des Problems.

\subsection{EMPIRISCHE BEFUNDE}

Und hier sprechen nun die empirischen Befunde deutlich gegen die Möglichkeit, dass arbeitslose Personen über das Bürgerschaftliche Engagement wieder gesellschaftlich aktiv werden. In diesem Fazit stimmen die wichtigsten Studien überein. So kommt etwa der Freiwilligensurvey 1999 zu folgender Differenz: Die Quote der im Bürgerschaftlichen Engagement Aktiven beträgt in der Gesamtbevölkerung 35,5\%, bei Arbeitslosen in Westdeutschland 24,6\%. In Ostdeutschland beträgt die Differenz 28,4 \% zu 22,2 \%. Im Verhältnis betrachtet geben die SOEP-Daten für den gleichen Zeitraum ähnliche Unterschiede wieder. Beachtenswert ist jedoch ein wesentlicher Unterschied zwischen West- und Ostdeutschland ${ }^{1}$ : Erstens ist in Ostdeutschland die Differenz zwischen der Engagementquote der Gesamtbevölkerung und der der Arbeitslosen nicht ganz so deutlich wie in Westdeutschland. Zweitens besteht in Westdeutschland auch eine deutliche Differenz der Engagementquote der Nicht-Erwerbstätigen über 14 Jahre $(31,9 \%)$ und der Quote der Arbeitslosen (24,6 \%), die in Ostdeutschland nicht besteht $(23,2 \% \mathrm{zu}$ $22,2 \%)$. Auch diese nur schwache Differenz wird in den Daten des SOEP bestätigt.

Die Differenz der Engagementquoten von arbeitslosen Personen und erwerbstätigen Personen zeigt sich auch im internationalen Vergleich auf der Grundlage des European Value Survey als überwiegend stabil. ${ }^{2}$

\footnotetext{
1 Zu den Zahlen siehe die Zusammenstellung von Dathe (2005, S. 476).

2 Eine Ausnahme bilden südeuropäische Gesell-
} schaften (Abbildung 1). 
Die unterschiedliche Höhe der Engagementquoten schließt jedoch noch nicht per se aus, dass sich Arbeitslosigkeit positiv auf die Aufnahme eines Bürgerschaftlichen Engagements auswirkt. Dies lässt sich präzise nur dann feststellen, wenn der Zusammenhang von Arbeitslosigkeit und Engagement im individuellen lebenszeitlichen Verlauf gemessen wird. Marcel Erlinghagen (2000) hat dies anhand des SOEP-Datensatzes für den Zeitraum von 1992-1996 untersucht. Dabei hat er drei Forschungshypothesen überprüft: Erstens, die Vermutung, dass mit dem Eintritt der Arbeitslosigkeit ein Problem der biografischen Sinnorientierung entstehen könnte, das wiederum durch Aktivitäten im Bürgerschaftlichen Engagement aufgefangen werden könnte. Zweitens nimmt er an, dass über eine Engagementaktivität berufliche Kompetenzen aufrechterhalten werden könnten, die im Fall von Arbeitslosigkeit (wegen mangelnder Praxis) verloren gingen. Diese beiden Faktoren müssten sich zusammengenommen, drittens, in einem Anstieg der Wahrscheinlichkeit zeigen, dass Personen, die vor Kurzem arbeitslos geworden sind, sich Aufgaben im Bürgerschaftlichen Engagement suchen.

Obwohl Erlinghagens Thesen theoretisch plausibel sind, konnte er dafür anhand seiner Längsschnittanalyse keinerlei empirische Bestätigung finden: „Arbeitslosigkeit hat in den alten Bundesländern keinen statistisch nachweisbaren Einfluss auf die Wahrscheinlichkeit, ein Ehrenamt aufzunehmen. Dieser Befund ist geschlechtsunabhängig und wird auch von der Länge der Arbeitslosigkeitsphase(n) nicht beeinflusst“ (Erlinghagen 2000, S. 302).

Gegen Erlinghagens Befunde lassen sich trotzdem noch drei Argumente einwenden. Erstens war seine Analyse auf Westdeutschland beschränkt. Die Untersuchungen von Dathe zeigen jedoch, dass es eine Differenz zwischen Ost- und Westdeutschland zu geben scheint hinsichtlich unterschiedlicher Wirkungen von Nicht-Erwerbstätigkeit und Arbeitslosigkeit. Diese Differenz zwischen Ost- und Westdeutschland könnte zweitens mit unterschiedlichen subjektiven Relevanzen der Erwerbstätigkeit bzw. Arbeitslosigkeit in den beiden Regionen zusammenhängen. Die ebenfalls sehr geringe Engagementquote bei nicht-erwerbstätigen Ostdeutschen könnte durch eine sehr starke biografische Bedeutung der Erwerbsarbeit erklärt werden. Deshalb müsste drittens dem subjektiven und biografischen
Zusammenhang von Erwerbsarbeit und Lebenszufriedenheit genauer nachgegangen werden. ${ }^{3}$

Dies haben die Schweizer Autoren Stephan Meier und Alois Stutzer (2004) getan, indem sie fragten ,is volunteering rewarding in itself"? Um eine Antwort zu gewinnen, haben sie ebenfalls den Datensatz des SOEP herangezogen. Sie prüfen den Einfluss von bedeutenden Lebensereignissen (Scheidung, Verwitwung, Arbeitslosigkeit, Aufnahme eines Engagements) auf die Lebenszufriedenheit der Person. Sie können allgemein feststellen, dass Bürgerschaftliches Engagement einen positiven Effekt auf die Lebenszufriedenheit hat (Meier/Stutzer 2004, S. 9), während sich für Arbeitslosigkeit eine deutlich negative Auswirkung auf die Zufriedenheit nachweisen ließ. Auch Ostdeutsche wiesen im Untersuchungszeitraum (unmittelbar nach der Wende 1992) eine im Durchschnitt geringe Lebenszufriedenheit auf, die sich noch deutlicher zeigte, wenn sie eine freiwillige Aktivität aufgeben mussten (Meier/Stutzer 2004, S. 27f.).

Allerdings finden wir auch in dieser Untersuchung keine nachweisbaren $\mathrm{Zu}$ sammenhänge zwischen Bürgerschaftlichem Engagement und Arbeitslosigkeit. Allenfalls wäre die Vermutung angebracht, dass Arbeitslose, die ein Engagement ausüben, eine höhere Lebenszufriedenheit aufweisen als Arbeitslose, die sozial inaktiv sind.

Hinzu kommt, dass es sich um $\mathrm{Zu}$ sammenhangsanalysen auf der Grundlage von „Durchschnittswerten“ handelt, die im Grunde genommen keine Rückschlüsse auf individuelle Handlungsgründe zulassen. Wenn es bei Max Weber heißt, dass „soziales Handeln deutend zu verstehen und dadurch zu erklären sei“, müsste für den möglichen Zusammenhang von biografischer Orientierung, Lebenszufriedenheit, Bürgerschaftlichem Engagement und Arbeitslosigkeit auf den individuellen Handlungssinn zurückgegangen werden. Insofern lässt sich anhand der Untersuchungen von Erlinghagen und Meier/Stutzer nachweisen, dass bei Arbeitslosen im Durchschnitt zwar keine signifikant höheren Chancen der Aufnahme eines Engagements bestehen, aber trotzdem für individuelle Konfigurationen der Arbeitslosigkeit Gründe für ein Engagement vorliegen könnten, die eine tatsächliche Aufnahme einer freiwilligen Aktivität bedingen.

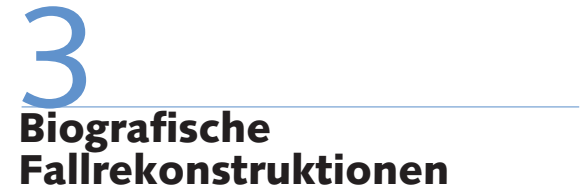

Die Handlungsgründe im Weberschen Sinne zu erschließen, war Ziel unserer empirischen Untersuchung zu „Bürgerschaftlichem Engagement und Politischer Kultur". 4 Dazu wurden insgesamt 114 Personen biografisch interviewt, von denen 79 bürgerschaftlich engagiert und 35 keiner freiwilligen sozialen Aktivität nachgegangen sind. Die Personen wurden bisher je zweimal biografisch interviewt, im Jahr 2002 und im Jahr 2005. Eine dritte Erhebung beginnt in diesem Jahr. Die Auswahl der Stichprobe ist nicht statistisch repräsentativ (keine Zufallsauswahl einer Grundgesamtheit), sondern bewusst quotiert, um bestimmte Engagementformen (im Recht, in der Kultur, in der Jugendarbeit, in der transnationalen sozialen Unterstützung) in ausreichender Zahl zu erheben. Anhand der biografischen Interviews konnten wir den objektiven Lebensverlauf (Bildungsverlauf, Erwerbsverlauf, Familienverlauf, Wohngeschichte) wie auch die familiären Herkunftsdaten (bis in die Großelternfamilie) genau erfassen. Insofern verfügen wir über Kenntnisse der objektiven $\mathrm{Zu}$ sammenhänge von Arbeitslosigkeitsepisoden und Engagementepisoden im Leben einer Person. Darüber hinaus kennen wir sowohl die Deutungen der Arbeitslosigkeitsphasen als auch die des ausgeübten Engagements. Durch retrospektive Erzählungen und den Panelcharakter der Interviews können wir Veränderungen der subjektiven Sichtweisen der Personen zuverlässig rekonstruieren.

Wir wollen in diesem Beitrag vier Fallvignetten aus Ostdeutschland genauer vorstellen und kontrastieren dabei je zwei bürgerschaftlich Engagierte und zwei NichtEngagierte. Wir finden ähnliche Kontraste auch in den Biografien von ehrenamtlich engagierten und nicht engagierten Frauen

3 Es wäre angesichts der ersten These von Erlinghagen erwartbar gewesen, dass er diesem Zusammenhang der subjektiven Befindlichkeit empirisch stärker nachgegangen wäre.

4 Die Studie wurde als Teilprojekt C4 im Rahmen des Sonderforschungsbereichs 580 an den Universitäten Halle und Jena durchgeführt. Eine ausführliche Darstellung der Studie findet sich bei Corsten et al. 2007. 
in Ostdeutschland, wollen uns aber aus darstellungspragmatischen Gründen auf die männlichen Erwerbs- und Engagementverläufe beschränken, die im Hinblick auf sozial-positionale Merkmale Ähnlichkeiten aufweisen. Dazu wählen wir jeweils einen Engagierten und einen Nicht-Engagierten aus der Akademiker-Schicht (Ingenieure) (Beispiele 3.1 und 3.3) und einen Engagierten und einen Nicht-Engagierten aus der Arbeiterschicht (Mechaniker) aus (Beispiele 3.2 und 3.4).

\subsection{BEISPIEL ENGAGIERTER AKADEMIKER}

Siegfried Nüssing 5 (Ingenieur, Interkulturarbeit) wurde 1959 in einer ostdeutschen Mittelstadt geboren. Seine Eltern stammten aus Westdeutschland und waren dort schon Mitglieder der kommunistischen Partei. Über ein technisches Abitur mit Berufsausbildung und nach der Armeezeit nahm er ein Studium des Konstruktionswesens an einer Technischen Universität auf. Danach war er von 1986 bis 1993 als Programmierer, Assistent des Fachdirektors und als verantwortlicher Mitarbeiter in der Betriebsorganisation eines Kombinats der optischen Industrie bzw. nach der Wende in einem Optik-Technologiezentrum tätig. 1993 wird er arbeitslos. Nach einem halben Jahr Arbeitslosigkeit nimmt er eine Tätigkeit in der Versicherungsbranche an, die er nach fünf Monaten wieder aufgibt. Nach wiederum einem halben Jahr Arbeitslosigkeit bekommt er (bis 1998) eine ABMStelle im Interkulturbereich. Nach einer weiteren Zeit der Arbeitslosigkeit wird er im Herbst 1999 Referent für Bildung und internationale Beziehungen bei einem ostdeutschen Landessportverband. Seit 2003 ist er dort als Geschäftsführer tätig.

Nüssing nahm bereits kurz nach der Wende ein Bürgerschaftliches Engagement im Interkulturbereich auf, indem er eine deutsch-französische Freundschaftsgesellschaft gründete, aus der 1999 eine deutschfranzösische Städtepartnerschaft erwuchs. Die Arbeitslosigkeitsphasen nutzt Nüssing intensiv, um die von ihm ins Leben gerufenen bürgerschaftlichen Initiativen voranzubringen. Er kann darüber wiederum Netzwerke mit sozialen Einrichtungen in seinem lokalen Umfeld aufbauen, die ihm langfristig einen Berufswechsel aus dem Bereich der optischen Industrie in den kulturellen und sozialen Dienstleistungssektor ermöglichen.
Im Fall Siegfried Nüssing zeigt der objektive Verlauf somit schon eine enge Verbindung zwischen einem Bürgerschaftlichen Engagement und der Bewältigung von Arbeitslosigkeit. Wichtig zu beachten ist hier, dass Nüssing bereits vor seiner Arbeitslosigkeit eine freiwillige soziale Aktivität übernommen hat. Zum Zeitpunkt des Eintritts der Arbeitslosigkeit verfügt Nüssing somit über Bildungskapital (Universitätsabschluss, Berufserfahrung), aber auch durch sein Engagement im Interkulturbereich über soziales und kulturelles Kapital. Auf der Grundlage dieser sozialen Ressourcen wird Arbeitslosigkeit nicht als Bedrohung wahrgenommen. Er fühlt sich deswegen auch nicht gezwungen, in Erwerbstätigkeiten dauerhaft aktiv zu sein, die aus seiner Sicht unattraktiv sind.

Aus der Perspektive der oben zitierten quantitativen Lebenslaufforschung ließe sich gegenüber dem Befund aus dem Fall Nüssing einwenden, dass sich der Einfluss der Arbeitslosigkeit auf Bürgerschaftliches Engagement und umgekehrt die positive Rückwirkung des Engagements auf die Wiederaufnahme einer Erwerbstätigkeit und sogar auf den Transfer von beruflichen Kompetenzen durch das hohe Bildungskapital erklären ließe. Seine Bildungsressourcen verschaffen ihm einen Spielraum im Hinblick auf die sozialen Formen, von denen er sich „aktivieren“ lässt.

Wir finden solche Fälle positiver Rückwirkung aber auch bei Personen mit einfachen Berufsausbildungen, wie die nächste Fallvignette zeigen soll.

\subsection{BEISPIEL ENGAGIERTER ARBEITER}

Nino Thomas (Traktorenschlosser, Schöffe) wurde 1970 in einer ostdeutschen Kleinstadt im ländlichen Milieu geboren. Sein Vater ist Leiter einer landwirtschaftlichen Produktionsgenossenschaft und Bürgermeister des Orts. Nach dem Besuch der Polytechnischen Oberschule (POS) absolviert er von 1987 bis 1989 eine Ausbildung zum Landmaschinen- und Traktorenschlosser. Diese Ausbildung erweist sich nach der Wende für ihn als praktisch wertlos. Zwischen 1990 und 1994 jobbt er in verschiedenen Vertretertätigkeiten, bis er 1994 über eine Tätigkeit als Angestellter und Busfahrer für ein Reisebüro beginnt, selbst Busreisen für Jugendliche zu organisieren. Darüber wechselt er in den Bereich der Jugendarbeit. Dort ist er ab 1996 zunächst als Streetworker, als Organisator für
Jugendreisen und dann als Betreuer in der sozialpädagogischen Familienhilfe tätig. Ab 1998 beginnt er über die Abendschule eine Berufsausbildung zur staatlich anerkannten Fachkraft für soziale Arbeit, danach ab 2003 eine Ausbildung zum systemischen Familientherapeuten.

Nino Thomas ist seit der Jugend vielfältig engagiert. Er beginnt als Junger Sanitäter, geht nach der Wende zum Jungen Roten Kreuz, in die Freiwillige Feuerwehr, in einen Gartenverein, ist nach der Geburt seiner Tochter (1997) bei einer Kindervereinigung tätig, beim Arbeiter-SamariterBund und als Schöffe ehrenamtlich aktiv. Ähnlich wie Nüssing kann Nino Thomas soziale Netzwerke vor Ort nutzen. Dies zeigt sich vor allem darin, dass er zwischen seinen Aktivitäten im Notfallwesen und als Reiseleiter/Busfahrer Verknüpfungen herstellen kann, die ihm ermöglichen, eine Art sozialen Dienst für Jugendreisen im ländlichen Raum zu etablieren. Er ist dabei von dem Motiv geleitet, sich in die Dinge und Prozesse, die ihn umgeben und ihn betreffen, aktiv einzumischen.

Die Fälle Nüssing und Thomas weisen mehrere Parallelen auf. Erstens haben wir es mit Söhnen von Vätern zu tun, die in der DDR eine einflussreiche Stellung besaßen. Zweitens haben beide schon frühzeitig in ihrer Biografie soziale Funktionen und Ehrenämter übernommen. Dieses soziale Kapital kommt ihnen im Fall von Arbeitslosigkeitsepisoden zugute. Drittens haben sie von ihren Vätern eine loyale Haltung gegenüber Gemeinschaften übernommen, die sie gegenüber den Aktivierungsimpulsen der Gesellschaft tendenziell offen sein lässt.

\subsection{BEISPIEL NICHT ENGAGIERTER AKADEMIKER}

Henry Bucht (Ingenieur) wird 1949 in einer Kleinstadt geboren, die in einer ostdeutschen Mittelgebirgsregion liegt. In der Familie leben insgesamt sieben Kinder. Der Vater ist Lehrer. Die Mutter arbeitet im späteren Lebensalter als Hortnerin und nach der Wende als Stadtführerin. Ähnlich wie Siegfried Nüssing wählt Henry Bucht nach Beendigung der POS eine Berufsausbildung (EDV-Lehre), die mit einem technischen Abitur abschließt. Danach studiert er zwischen 1969 und 1973 Mathematik.

\footnotetext{
5 Die Namen der Befragten wurden selbstverständ-
} lich geändert. 
Unmittelbar nach dem Studium bekommt er eine Stelle als wissenschaftlicher Mitarbeiter in einem Chemiekombinat, in dem er elf Jahre (bis 1984) tätig ist. Danach wechselt er auf eine Stelle als Gruppenleiter (ohne disziplinarische Verantwortung) in der EDV-Abteilung eines Elektromotorenwerks. Nach der Wende nimmt er eine Tätigkeit im Vertrieb von Computer-Software auf und macht sich 1994 selbstständig mit einer eigenen Firma, die Computer und Software an Firmen verkauft. Allerdings ist nach Buchts Erfahrungen diese Form der Selbstständigkeit ökonomisch nicht sehr einträglich.

Henry Bucht ist in seiner Biografie niemals im eigentlichen Sinn freiwillig sozial tätig gewesen. Auch heute nicht, obwohl er aufgrund der geringen Auftragslage seiner Firma im Prinzip sowohl über genügend Zeit als auch über die Chance zur freien Zeiteinteilung verfügt. In die Nähe eines Bürgerschaftliches Engagements kommt seine Mitgliedschaft in einer Singspielgruppe zu DDR-Zeiten, aus der er aber später ausscheidet. Gegenwärtig hält sich Bucht mit einer eigenen Ein-Mann-Firma „über Wasser“. Er versucht, sich ein zweites „Standbein“ durch Dia-Vorträge über Stereophotographie von Landschaften aufzubauen. Sein Einkommen beziffert er mit unter $750 €$ monatlich. Bucht kompensiert drohende Arbeitslosigkeit durch individuelle Ausweichstrategien in prekäre Selbstständigkeit. Er fällt in eine Grauzone zwischen ungewisser Beschäftigungssituation und working poor.

\subsection{BEISPIEL NICHT ENGAGIERTER ARBEITER}

Der Taxifahrer Maik Rothändel (gelernter KFZ-Mechaniker) wurde 1971 in einer ostdeutschen Stadt mittlerer Größe geboren. Nach Scheidung der Eltern wächst er einige Jahre bei den Großeltern auf. Der
Großvater ist Fernmeldemechaniker, der Stiefvater ist Videotechniker. Sein leiblicher Vater betreibt nach der Wende ein Taxiunternehmen. Maik Rothändel absolviert Ende der 1980er eine Lehre als Kfz-Mechaniker und Berufskraftfahrer. Nach der Wende ist er als Fahrer bei der Post angestellt. Nach einem Autounfall unter Alkoholeinwirkung wechselt er als Aushilfskraft in einen Kneipenbetrieb. Seit 1994 arbeitet er als Taxifahrer, zuletzt in der Firma seines leiblichen Vaters. 1995 wird seine Tochter geboren. Rothändel und die Mutter des Kindes leben nicht-ehelich zusammen. Sie arbeitet als Krankenschwester.

Wie Henry Bucht ist auch Maik Rothändel in seiner Biografie noch nie ehrenamtlich tätig gewesen. Ähnlich wie im Fall Bucht ließe sich das mit der spezifischen Berufssituation erklären. Als Selbstständiger oder als Taxifahrer hat man rund um die Uhr zu tun. Allerdings erwähnen die beiden interviewten Personen, dass sie relativ frei über ihre Zeit verfügen können. Seine Freizeit verbringt Rothändel z. B. mit Kumpels beim Fußball, wobei er seine Tochter mit zu Spielen seines Lieblingsvereins nimmt.

Bucht und Rothändel sind in ihren Erwerbsbiografien jeweils nur kurzzeitig arbeitslos. Sie kompensieren diese Phase jedoch auf deutlich andere Weise als Nüssing und Thomas. Henry Bucht tendiert zu individualistischen Strategien, indem er in Form der Selbstständigkeit die Risiken drohender Arbeitslosigkeit und die Verantwortung für ein Existenz sicherndes Einkommen ganz auf sich nimmt. Maik Rothändel ist durch familiäre Netzwerke gestützt. Sein Großvater verhilft ihm zunächst zu einer Tätigkeit als Fahrer bei der Post, heute ist Rothändel im Unternehmen seines leiblichen Vaters als Taxifahrer tätig und seine Lebenspartnerin verfügt als Krankenschwester über eine sichere Stellung und ein gesichertes Einkommen.

\section{Tabelle 1: Erwerbsbiografische Nutzung des Bürgerschaftlichen}

Engagements als ...

\begin{tabular}{|c|c|c|c|c|c|c|c|}
\hline & & & $\begin{array}{c}\text {...Beziehungs- } \\
\text { kapital }\end{array}$ & $\begin{array}{c}\text {...Überbrückung } \\
\text { unbezahlter } \\
\text { Projektphasen }\end{array}$ & $\begin{array}{l}\text {...Komplement } \\
\text { der Erwerbs- } \\
\text { arbeit im } \\
\text { Ruhestand }\end{array}$ & $\begin{array}{c}\text {...Kein } \\
\text { Erwerbs- } \\
\text { bezug }\end{array}$ & Gesamt \\
\hline \multirow{2}{*}{ Ost } & \multirow{2}{*}{ Arbeitslos } & Nein & 1 & - & 10 & 13 & 24 \\
\hline & & $\mathrm{Ja}$ & 9 & - & 1 & 3 & 13 \\
\hline \multirow{2}{*}{ West } & \multirow{2}{*}{ Arbeitslos } & Nein & 1 & 1 & 6 & 31 & 39 \\
\hline & & $\mathrm{Ja}$ & - & 2 & - & 1 & 3 \\
\hline Gesamt & & & 11 & 3 & 17 & 48 & 79 \\
\hline
\end{tabular}

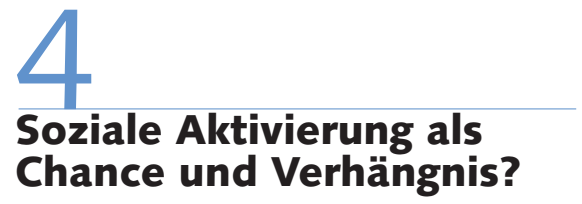

Die Erwerbsituation in Ostdeutschland ist bis heute durch drei markante Unterschiede gekennzeichnet: (a) eine höhere Bedeutung der Erwerbstätigkeit in den subjektiven Lebensorientierungen der Bevölkerung; (b) höhere Arbeitslosigkeitsquoten und (c) geringere Nicht-Erwerbstätigkeitsquoten von Frauen.

Mit diesen drei Faktoren lassen sich die Differenzen der Engagementquoten zwischen Ost- und Westdeutschland in makrosoziologischer Perspektive weitgehend erklären. Der höhere Anteil an Arbeitslosen und die höhere Bewertung der Erwerbsarbeit erklärt den geringeren Anteil von bürgerschaftlich Engagierten in Ostdeutschland; der höhere Anteil von erwerbstätigen Frauen die geringe Differenz der Engagementquoten zwischen nicht-erwerbstätigen und arbeitslosen Personen in Ostdeutschland - bzw. umgekehrt: Die höhere Quote des Engagements bei Nicht-Erwerbstätigen in Westdeutschland - beruht zu großen Teil auf dem Ehrenamt von nicht-berufstätigen Frauen im mittleren Lebensalter.

So schlüssig diese makroskopischen Zusammenhänge auch sein mögen, aus ihnen folgt nicht notwendigerweise die Irrelevanz des Bürgerschaftlichen Engagements im Fall von Arbeitslosigkeit. Mit den Fallbeispielen 3.1 (Nüssing) und 3.2 (Thomas) konnten wir Konfigurationen zeigen, in denen Engagement dem Aufbau sozialer Netzwerke diente, die als soziales Kapital bei Arbeitslosigkeit - insbesondere beim Übergang in neue Felder der Erwerbsaktivität - genutzt werden.

In unserer Stichprobe tritt diese Nutzung des Ehrenamts in Ostdeutschland deutlich häufiger auf als in Westdeutschland, wie die Tabelle 1 zeigt.

Auch wenn diese Befunde in quantitativer Hinsicht nicht als repräsentativ gewertet werden können, so vermitteln sie doch wichtige Anhaltspunkte, die in künftigen Studien genauer verfolgt werden sollten:

Sie zeigen die Bedeutung von spezifischen erwerbsbiografischen Re-Aktivierungsstrategien, die in quantitativen Studien (bisher) nicht angemessen nachvollzogen werden konnten. Anhand der vier beispielhaft ausgewerteten Fälle konnten 
wir nachweisen, über welche individuellen Aktivierungsstrategien soziales Kapital aufgebaut wird, das bei der Platzierung auf dem Arbeitsmarkt verwendet werden kann. Das Beispiel 3.3 (Bucht) zeigt eine individualistische Strategie der Aktivierung, die (vermutlich sogar bewusst) auf Abhängigkeiten von sozialen Netzwerken (im öffentlichen oder familiären Raum) verzichtet. Der Nicht-Engagierte Rothändel (3.4) kann fehlende soziale Netzwerkbeziehungen zum öffentlichen Raum durch familiär vorhandenes soziales Kapital kompensieren. In den Beispielen 3.1 und 3.2 sehen wir, wie über Bürgerschaftliches Engagement soziales Kapital im Raum der lokalen Öffentlichkeit aufgebaut wird, das zur erwerbsbiografischen Re-Aktivierung nach Phasen der Arbeitslosigkeit genutzt werden kann. Bedeutsam erscheint dabei vor allem, dass dieses in der Regel zeitlich unabhängig vom Eintritt einer akuten Arbeitslosigkeit erworben wurde.

Die erwerbsbiografische Selbst-Aktivierung über Bürgerschaftliches Engagement scheint somit auf den ersten Blick vorteilhaft für die von uns vorgestellten ostdeutschen Akteure gewesen zu sein. Un- tergründig bestärken ihre Reaktionen aber womöglich eine gesellschaftliche Spaltung in sogenannte „Freiwillig Aktive“ und „Inaktive“. Zur Autonomie einer erwerbstätigen Person gehört jedoch auch die Chance, angebotene Aktivitätsmöglichkeiten zurückweisen zu können - wie im Beispiel 3.1 (Nüssing) sichtbar wurde. Dies können aber wiederum nur diejenigen, die über ausreichend soziale, kulturelle oder ökonomische Ressourcen verfügen. Diese (neue) soziale Ungleichheit kann durch eine gestiegene Aktivierung der Individuen nicht ausgeglichen werden.

\section{LITERATUR}

Corsten, M./Kauppert, M./Rosa, H. (2007): Quellen bürgerschaftlichen Engagements, Wiesbaden

Dathe, D. (2005): Bürgerschaftliches Engagement, in: Soziologisches Forschungsinstitut et al. (Hrsg.): Berichterstattung zur sozioökonomischen Entwicklung in Deutschland, Wiesbaden, S. 455-480

Erlinghagen, M. (2000): Arbeitslosigkeit und ehrenamtliche Tätigkeit im Zeitverlauf. Eine Längsschnittanalyse der westdeutschen Stichprobe des Sozio-oekonomischen Panels (SOEP) für die Jahre 1992 und 1996, in: Kölner Zeitschrift für Soziologie und Sozialpsychologie 2, S. 291-310 Gensicke, T./Picot, S./Geiss, S. (2006): Freiwilliges Engagement in Deutschland 1999-2004, Wiesbaden
Halman, L./Luijkx, R./Zundert, M. v. (2006): Atlas of European Values, Leiden

Keupp, H./Kraus, W./Straus, F. (2000): Civics matters: Motive, Hemmnisse und Fördermöglichkeiten bürgerschaftlichen Engagements, in: Beck, U. (Hrsg.): Zukunft von Arbeit und Demokratie, Frankfurt, S. 217-268

Lessenich, S. (2008): Die Neuerfindung des Sozialen, Bielefeld Meier, S./Stutzer, A. (2004): Is Volunteering Rewarding in Itself?, IZADiscussion Paper 1045, Zürich

Rosenbladt, B. v. (2000): Freiwilliges Engagement in Deutschland Freiwilligensurvey, Stuttgart 